\title{
The impact of nutritional counseling in the healthy diet score in type 1 and type 2 diabetes
}

\author{
Rodrigo Bastos Fóscolo*, Ann Kristine Jansen, Carolina Jovita Oliveira Dias \\ From 20th Brazilian Diabetes Society Congress \\ Porto Alegre, Brazil. 11-18 November 2015
}

\section{Background}

In the last decade in Brazil, the inadequate nutrition appears as the main risk factor for disability related chronic diseases.

\section{Objective}

To evaluate the impact of nutritional counseling in the healthy diet score in type 1 diabetes (T1D) and type 2 (DM2).

\section{Materials and methods}

Intervention study with 39 adult diabetic patients of both sexes after a year of individual clinical and nutritional monitoring, the multidisciplinary outpatient Diabetes League, 19 DM1 and DM2 20. During the research the patients were instructed to change their eating pattern according to the recommendations of the American Diabetes Association. We collected data from medical records at the first visit and one year after. Clinical, biochemical, anthropometric and food consumption were evaluated. Food consumption was assessed by a healthy diet score that classifies food into three categories of variables: Ideal (score 2), intermediate (score 1) and poor (score 0 ). The maximum score possible was 16, considering ideal: Daily consumption of foods rich in monounsaturated fatty acids, at least 2 servings of whole grains and 400 grams of vegetables fruits and vegetables; three times a week fish, at most once a week processed foods high in sodium, high in animal fat, fried foods and soft drinks. The score reliability test was satisfactory (Cronbach's alpha $=0.614$ ). Statistical analysis was performed with SPSS 17.0 software.

\section{Results}

At baseline the DM1 group's average score was 5.89 $( \pm 2.48)$ and DM2 $5.98( \pm 2.93)$. At end, both groups presented significant increase $(\mathrm{p}<0.05)$, and the average DM1

* Correspondence: CLINICAFOSCOLO@GMAIL.COM

UFMG, Belo Horizonte, Brazil 